\title{
Dissecting medial temporal lobe contributions to item and associative memory formation
}

\author{
Shaozheng Qin a,b,d,*, Mark Rijpkema a , Indira Tendolkar ${ }^{c}$, Carinne Piekema ${ }^{\text {a }}$, Erno J. Hermans ${ }^{\mathrm{a}}$, \\ Marek Binder ${ }^{\mathrm{e}}$, Karl Magnus Petersson ${ }^{\mathrm{a}}$, Jing Luo ${ }^{\mathrm{d}}$, Guillén Fernández ${ }^{\mathrm{a}, \mathrm{b}}$ \\ a Donders Institute for Brain, Cognition and Behaviour, Radboud University Nijmegen, 6500 HB Nijmegen, The Netherlands \\ ${ }^{\mathrm{b}}$ Department of Neurology, Radboud University Nijmegen Medical Center, 6500 HB Nijmegen, The Netherlands \\ ${ }^{c}$ Department of Psychiatry, Radboud University Nijmegen Medical Center, 6500 HB Nijmegen, The Netherlands \\ ${ }^{\mathrm{d}}$ Key Laboratory of Mental Health, Institute of Psychology, Chinese Academy of Sciences (CAS), 100101 Beijing, China \\ e Psychophysiology Unit, Jagiellonian University, Cracow, Poland
}

\section{A R T I C L E I N F O}

\section{Article history:}

Received 10 October 2008

Revised 15 January 2009

Accepted 23 February 2009

Available online 9 March 2009

\section{Keywords:}

Episodic memory

Hippocampus

Parahippocampal cortex

Perirhinal cortex

Prefrontal cortex

\begin{abstract}
A B S T R A C T
A fundamental and intensively discussed question is whether medial temporal lobe (MTL) processes that lead to non-associative item memories differ in their anatomical substrate from processes underlying associative memory formation. Using event-related functional magnetic resonance imaging, we implemented a novel design to dissociate brain activity related to item and associative memory formation not only by subsequent memory performance and anatomy but also in time, because the two constituents of each pair to be memorized were presented sequentially with an intra-pair delay of several seconds. Furthermore, the design enabled us to reduce potential differences in memory strength between item and associative memory by increasing task difficulty in the item recognition memory test. Confidence ratings for correct item recognition for both constituents did not differ between trials in which only item memory was correct and trials in which item and associative memory were correct. Specific subsequent memory analyses for item and associative memory formation revealed brain activity that appears selectively related to item memory formation in the posterior inferior temporal, posterior parahippocampal, and perirhinal cortices. In contrast, hippocampal and inferior prefrontal activity predicted successful retrieval of newly formed inter-item associations. Our findings therefore suggest that different MTL subregions indeed play distinct roles in the formation of item memory and inter-item associative memory as expected by several dual process models of the MTL memory system.
\end{abstract}

(C) 2009 Elsevier Inc. All rights reserved.

\section{Introduction}

The ability to remember single items and to bind different aspects of an experience into a coherent episode are key features of episodic memory (Tulving, 1983, 2002). However, a fundamental and debated question is whether neural processes leading to a memory for individual items (i.e. non-associative or item memory) differ from processes underlying memory formation for associations among disparate elements of an experience (i.e. associative or relational memory).

One line of research suggests that distinct medial temporal lobe (MTL) subregions with different architecture and connectivity like the hippocampus and the parahippocampal gyrus, which is covered by entorhinal, perirhinal, and parahippocampal cortices, support distinct mnemonic operations along the dimension of non-associative item

\footnotetext{
* Corresponding author. Centre for Cognitive Neuroimaging, Donders Institute for Brain, Cognition and Behaviour, Radboud University Nijmegen, P.O. Box 9101, 6500 HB Nijmegen, The Netherlands. Fax: +31243610989.

E-mail address: shaozheng.qin@donders.ru.nl (S. Qin).
}

and associative memory (Brown and Aggleton, 2001; Eichenbaum et al., 1994, 2007; Yonelinas et al., 2002; Davachi, 2006). These twocomponent models distinguish between the hippocampal contributions critically important for associative memories and the perirhinal contributions sufficient for item memory. More recently, Eichenbaum et al. (2007) extended their model into a three-component model in which the parahippocampal cortex contributes to associative memory by coding contextual information while the perirhinal cortex supports single item memory and the hippocampus supports associative memory by binding item(s) with relevant contextual information. Several lines of evidence support such a division of labor especially during memory retrieval, because item recognition memory is correlated with activation reductions in the perirhinal cortex while associative retrieval or recollection is correlated with a hippocampal activity increase (Weis et al., 2004; Gonsalves et al., 2005; Henson, 2005; Montaldi et al., 2006; Tendolkar et al., 2008; for reviews see Diana et al., 2007; Eichenbaum et al., 2007).

Contrary to this divided labor account, another line of research suggests that MTL subregions work together in a cooperative and complementary way and thus differences found between hippocampal 
and parahippocampal contributions are rather related to memory strength instead of item and associative memories (Squire et al., 2007). In such a unitary MTL model, the hippocampus at the top of the hierarchy might be associated with stronger memories than the parahippocampal region, which is subordinate and thus associated with weaker memories. Several lines of evidence support this memory strength scenario, especially during memory formation (Rutishauser et al., 2006; Tendolkar et al., 2007), because the parahippocampal region in particular the perirhinal cortex may support weaker memories for both items and associations. For example, activity in the hippocampus and the perirhinal cortex correlates with subsequent memory strength (Shrager et al., 2008), and the perirhinal cortex appears also involved in associative memory formation (Jackson and Schacter, 2004; Kirwan and Stark, 2004; Uncapher et al., 2006; Staresina and Davachi, 2006, 2008).

Taken together, there are conflicting findings regarding the question whether hippocampal and parahippocampal contributions to memory formation are distinct between processes for item and associative memories or not. To tackle this issue, we developed a new design for our event-related fMRI study of memory formation mitigating two central problems. Firstly, to get the best possible separation of brain activity related to item and associative memory formation, we aimed at separating these processes not only behaviorally and anatomically, but also temporally. Therefore, we presented items sequentially with an intra-pair delay of several seconds and participants could not form task relevant associations when encoding the first event, because the second one was not yet known to them. Secondly, differences in memory strength between the item and the associative memory formation conditions may have been reduced. To do so, we increased the difficulty of the item recognition memory test by pairing each item to be memorized with an item of the same semantic category as a lure in the item recognition memory test. Furthermore, encoding trails (inter-item associations) whose constituents were recognized with similar levels of confidence (i.e. memory strength) in the item recognition memory test, were further sorted into the unsuccessful (i.e. item memory) and the successful associative memory formation conditions, according to memory performance of the final associative recognition memory test. If we find similar item- and association-related subsequent memory effects in the hippocampus and the perirhinal cortex, our data would be in line with a unitary MTL model. However, if we find different subsequent memory effects despite similar memory confidence, our data would rather support a process dissociation in line with the two- or three-component models of the MTL mentioned above.

\section{Materials and methods}

\section{Participants}

Twenty-four young healthy university students (mean age $=23.8 \pm$ 3.5 years; 8 males) without any neurological or psychiatric history and normal/corrected-to-normal vision participated in this experiment. Participants gave written informed consent according to the local ethics committee and the declaration of Helsinki. We excluded behavioral and fMRI data from five participants: Four participants showed poor memory performance (mean correct rate $<0.54$ in either the item or the associative recognition memory test) and one participant caused excessive movement artifacts (larger than $6 \mathrm{~mm}$ ).

\section{Stimuli}

We selected 824 color photographs of different objects depicted on a black background and covering a wide range of semantic categories from a commercially available database (Hemera Photo-Objects; http://www.hemera.com/). This stimulus set contained two exemplars of each object category (e.g. two hammers, two dogs etc.). One exemplar of each category was used as a study item and the other one as the lure for item recognition memory test. The assignment to the study and the lure set was balanced across participants. For each set, we created 200 pairs of semantically unrelated objects for the study phase, again balanced across participants, and six additional pairs for a pre-scan training session. In a separate pilot study, all pairs were screened by three additional participants to avoid semantically related objects being paired together. The 34 pairs in which at least one subject indicated initially a semantic relationship were modified so that no semantic relationship was detected in a re-test

\section{Task procedure}

The experiment consisted of a paired-associate learning phase and two subsequent memory tests. During the study phase, fMRI scans were acquired while participants memorized 200 pairs of unrelated objects. Each trial started with the first object ('Event-1') presented at the center of the screen for $2 \mathrm{~s}$, followed by a variable delay period (ranging from 6 to $10 \mathrm{~s}$ in steps of $1 \mathrm{~s}$; average $8 \mathrm{~s}$ ), and then followed by the second object ('Event-2') presented at the center of the screen for $2 \mathrm{~s}$ (see Fig. 1). Trials were separated by an inter-trial-interval (ITI) ranging from 6 to $10 \mathrm{~s}$ (in steps of $1 \mathrm{~s}$; average $8 \mathrm{~s}$ ). Participants were explicitly instructed to memorize objects and object-pairs for two subsequent memory tests. To make sure that each pair was perceived as
Paired-associate learning

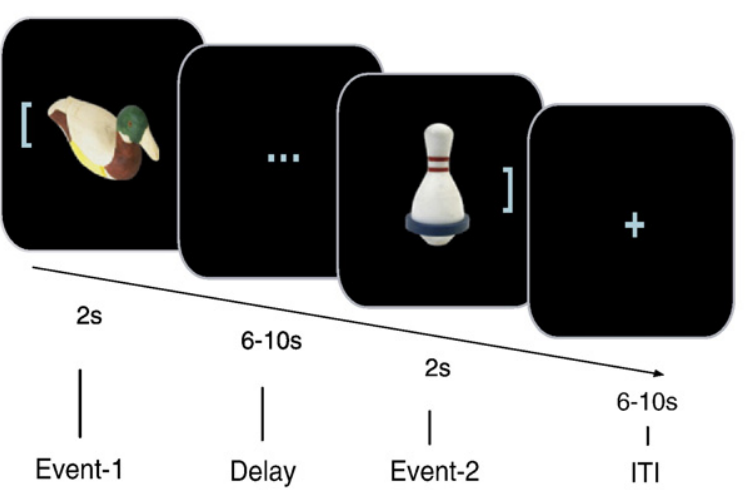

\section{Subsequent memory tests}

$\sim 3$ h later

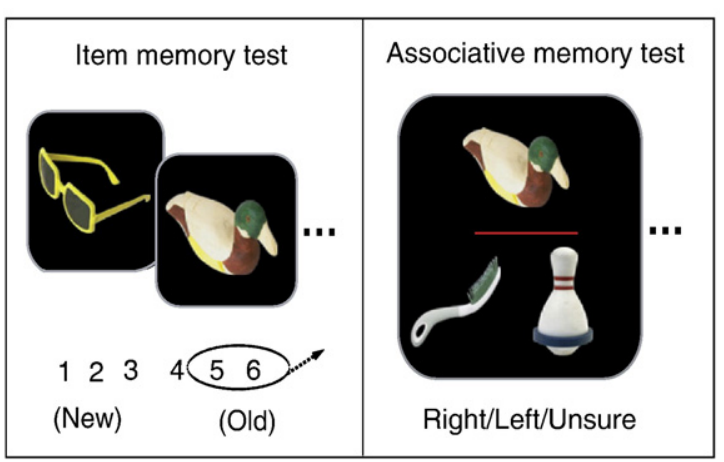

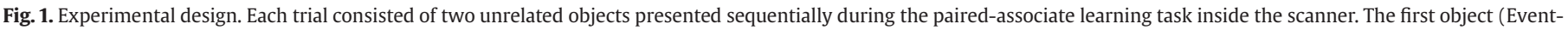

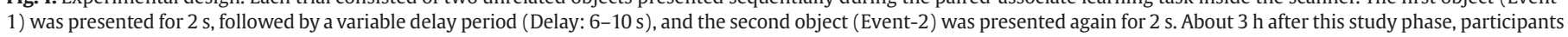
performed item and associative recognition memory tests outside the scanner (see Task procedure for more details). 
Table 1

Averaged number of trials on three conditions of interest.

\begin{tabular}{llll}
\hline & Forgotten & Item & Association \\
\hline Mean (SEM) & $34.47(1.85)$ & $21.26(1.64)$ & $40.21(3.15)$ \\
\hline
\end{tabular}

Note. 'Forgotten': Neither the first event nor the second event was later remembered in the item recognition memory test; 'Item': Both events or items were recognized, but incorrectly associated with the second event; 'Association': Both events and their association were correctly recognized.

such within a continuous stream of sequentially presented objects, we implemented two features: (1) The first object of each pair was presented together with an open bracket on its left hand side and the second one with a closed bracket on its right hand side; (2) a fixation cross was presented during the ITI between pairs and three horizontally grouped dots were presented during the intra-pair delay period. Thirty null events of $6 \mathrm{~s}$ duration and showing a central fixation cross were randomly inserted between trials. The whole study phase was divided into two runs. Prior to scanning, participants performed additional six trials in order to familiarize with task procedures.

About $3 \mathrm{~h}$ after the study phase, participants were asked to perform two memory tests outside the scanner. First, an item recognition memory test was given with 400 previously studied objects (all items from both Event 1 and 2) randomly intermixed with 400 related objects as lures. Participants were asked to judge whether each object was an old (studied) or a new (unstudied) object on a 6-point confidence rating scale $(1=$ absolutely sure it was a new object; $2=$ somewhat sure it was a new object; $3=$ guessing it was a new object; $4=$ guessing it was an old object; $5=$ somewhat sure it was an old object; $6=$ absolutely sure it was an old object). This task was self-paced with a minimal presentation time of $2 \mathrm{~s}$ and a maximal one of $6 \mathrm{~s}$. Objects presented as the first event during the study phase that received a 6 or 5 rating in the item recognition memory test served as cue-objects for a subsequent associative recognition memory test. In this test, two-alternative forced choice paradigm (2-AFC) was used. That is, each cue-object was shown together with two studied objects presented as a second event during the study phase (see Fig. 1). One of the two objects was previously paired with the cue-object; the other one was presented with a different object and served as a lure. This approach may bear a potential risk of reducing the power to detect associative memory formation, because the correct association could have been picked by exclusion. However, it appears more likely that most decisions are made on the basis of two actually present items instead of one absent and one present item and thus this approach is widely used (Sperling et al., 2002, 2003; Dickerson et al., 2005; Chua et al., 2007; Qin et al., 2007; Hales et al., 2009; Miller et al., 2008). Participants were informed that all objects were shown in the study session as well as in the single item recognition memory test to ensure that the decision about the correct association was not based solely on familiarity. The position of the correct object on the screen was counterbalanced to the right and left side (see Fig. 1). This task was also self-paced with a minimal presentation time of $3 \mathrm{~s}$ and a maximal one of $8 \mathrm{~s}$. Participants were asked to indicate by an appropriate button press which object had been associated with the cue-object during the study phase. Given the experimental design, the number of associations in the associative recognition memory test was limited to 100 trials, because half of the second event objects served as lures. However, all participants had more than 100 item hits related to the first event with a ' 5 ' or ' 6 ' rating in the item recognition memory test and thus we selected randomly 100 objects for the associative memory test out of these hit trials. To exclude trials potentially contaminated by guessing, participants were instructed to give an unsure response if they did not remember which association was the correct one.

\section{fMRI data acquisition}

During MRI scanning, whole brain T2*-weighted EPI-BOLD fMRI data were acquired with a Siemens Sonata 1.5 T MR-scanner using an ascending slice acquisition sequence (35 axial slices, volume $\mathrm{TR}=2.75 \mathrm{~s}, \mathrm{TE}=40 \mathrm{~ms}, 90^{\circ}$ flip-angle, slice-matrix size $=64 \times 64$, slice thickness $=3.0 \mathrm{~mm}$, slice gap $=0.5 \mathrm{~mm}$, field of view $=224 \mathrm{~mm}$ ). High-resolution structural images $\left(1 \times 1 \times 1 \mathrm{~mm}^{3}\right)$ were acquired using a T1-weighted MP-RAGE sequence (volume TR $=2250 \mathrm{~ms}$, $\mathrm{TE}=3.93 \mathrm{~ms}, 15^{\circ}$ flip-angle, 176 sagittal slices, slice-matrix size $=256 \times 256$, slice thickness $=1 \mathrm{~mm}$, field of view $=256 \mathrm{~mm}$ ).

\section{fMRI data analysis}

Image pre-processing and statistical analysis was performed using SPM5 (www.fil.ion.ucl.ac.uk/spm). The first five volumes of each participant's EPI-data were discarded to allow for T1 equilibration. The functional EPI-BOLD contrast images were realigned and the mean of functional images was coregistered to the structural MR image using mutual information optimization. Subsequently, functional images were slice-time corrected, spatially normalized, resampled to create $3 \mathrm{~mm}$ isotropic voxels and transformed into a common stereotactic space, as defined by the SPM5 MNI T1 template, as well as spatially filtered by convolving the functional images with an isotropic 3D Gaussian kernel (8 mm FWHM).

Encoding trials were sorted into several categories (see Table 1) based on subsequent memory performance. Transient neural activity associated with successful formation of associative and item memory is at issue. Therefore, we created separate regressors to assess the activity of the three temporal components in each trial (Event-1, Delay, Event-2) as a function of subsequent memory performance, and convolved these with the canonical hemodynamic response function. The onset and offset of the delay period vector were spaced apart from the first and the second event vectors by $2 \mathrm{~s}$ to increase the orthogonality of the regressors (Murray and Ranganath, 2007; Hannula and Ranganath, 2008). This approach resulted in an averaged co-linearity estimate of all pairs of regressors of less than 0.32 .

In relation to the item recognition memory test, encoding trials in which both the first event and the second event received a ' 1 ', ' 2 ' or ' 3 ' rating were regarded as 'forgotten', encoding trials in which either one of the two events received a ' 4 ' rating were regarded as 'unsure', and encoding trials in which the first event received a ' 5 ' or ' 6 ' rating were regarded as 'Event-1 hits' (see Behavioral results). Trials in which the first event was remembered were subdivided into the following categories depending on associative recognition memory test: (1) Single items remembered ('item'): Both events (or items) were recognized, but incorrectly associated with the second event; (2) Associations remembered or successful associative memory formation ('association'): Both events and their association were correctly recognized; (3) Trials in

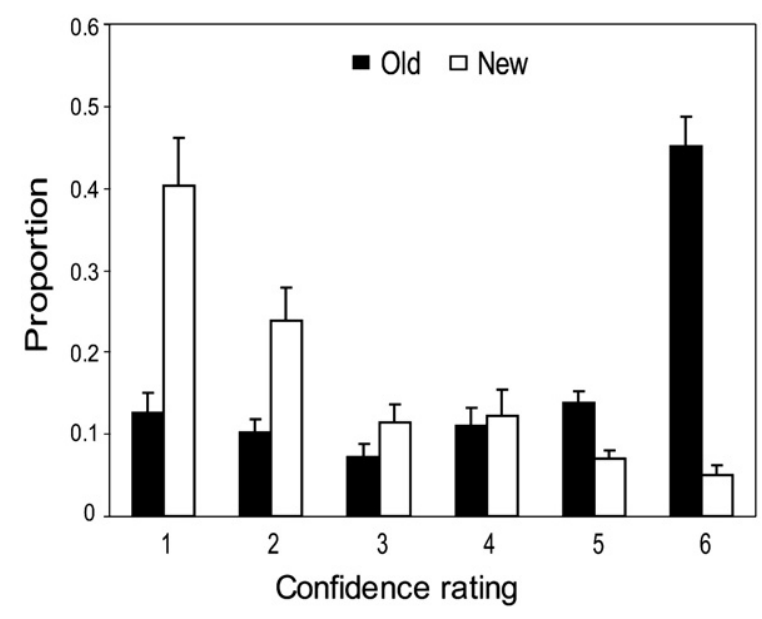

Fig. 2. Behavioral performance in item recognition memory test. Distributions of mean hit and false alarm rates: Mean (SEM) proportions of responses are shown on the $y$-axis and confidence ratings (' 1 ': absolutely new; ' 6 ': absolutely old) are depicted on the $x$-axis. 
Table 2

Brain activations derived from subsequent memory analyses.

\begin{tabular}{|c|c|c|c|c|c|c|}
\hline \multirow[t]{2}{*}{ Brain region } & \multirow[t]{2}{*}{ BA } & \multirow{2}{*}{$\begin{array}{l}\text { Cluster } \\
\text { size }\end{array}$} & \multirow[t]{2}{*}{ T score } & \multicolumn{3}{|l|}{ MNI } \\
\hline & & & & $x$ & $y$ & $z$ \\
\hline \multicolumn{7}{|l|}{ Event-1: Item vs. forgotten } \\
\hline Posterior inferior temporal cortex & R $19 / 37$ & 61 & $4.94^{\mathrm{a}}$ & 48 & -66 & -9 \\
\hline Posterior fusiform gyrus & L 37 & 25 & $3.68^{\mathrm{a}}$ & -51 & -57 & -9 \\
\hline Posterior parahippocampal cortex & R 19 & 17 & $4.16^{\mathrm{b}}$ & 33 & -42 & -21 \\
\hline \multirow[t]{2}{*}{ Rhinal cortex } & R 20 & 6 & $3.70^{c}$ & 42 & -15 & -27 \\
\hline & L 20/36 & 14 & $3.88^{\mathrm{c}}$ & -39 & -18 & -21 \\
\hline \multicolumn{7}{|l|}{ Event-2: Association vs. item } \\
\hline Hippocampus & L $35 / 36$ & 15 & $4.56^{\mathrm{b}}$ & -33 & -24 & -21 \\
\hline Inferior prefrontal cortex & L $47 / 11$ & 17 & $4.05^{c}$ & -48 & 30 & -6 \\
\hline
\end{tabular}

Note. A threshold $p<0.001$ (uncorrected) was used in the whole brain volume search and only clusters with 6 or more significant voxels are reported.

Event-1, time-locked to the onset of the first event; Event-2, time-locked to the onset of the first event; L, left; R, right; BA, Brodmann area; MNI, MNI coordinates (SPM5).

a Cluster level $p<0.01$ the whole brain volume corrected.

b Cluster level $p<0.05$ small volume correction (SVC) corrected for the MTL ROIs.

c Voxel level $p<0.001$ the whole brain volume uncorrected.

which the association was 'remembered' correctly, but the second event was not recognized were put into a separate bin, because they are likely contaminated by guessing and different levels of memory strength for the two constituents of an association. Also, all remaining trials were included into a condition 'of no interest'. In addition, the realignment parameters were included to account for movement-related variability, and the low-level null events were modeled separately. The data was analyzed using the general linear model and statistical parametric mapping (Friston et al., 1995). Statistical analysis in SPM5 included highpass filtering (128s) to account for various low-frequency effects, global intensity normalization, and serial correlations correction using autoregressive $A R(1)$ model.

The relevant contrast parameter images generated at the single subject level were submitted to the second-level group analysis. At the group level, the relevant contrasts between conditions were computed using a two (onsets: Event-1, Event-2) by three (Memory: Forgotten, Item, Association) analysis of variance (ANOVA). In the whole brain analysis, results from the random effects analyses were initially thresholded at $p<0.001$ (uncorrected), and the cluster size statistics were used as the test statistic. Unless otherwise specified, only clusters significant at $p<0.05$ (corrected for multiple nonindependent comparisons; Worsley et al., 1996) are reported together with the MNI coordinates of their local maximum. Given our clear hypothesis regarding the MTL, across both hemispheres two separate anatomical regions of interest (ROI) were created one for the hippocampus and the other for the PHG. For this purpose, individual, hand-drawn anatomical ROIs (i.e. on the basis of a standardized protocol; Pruessner et al., 2000, 2002) were normalized and averaged and then used as search volume for the small volume correction (SVC). Furthermore, to characterize the response patterns in brain regions involved in item and associative memory formation, the beta values separated for each condition were extracted from these regions using MarsBar (http://marsbar.sourceforge.net/; Brett et al., 2002).

\section{Results}

\section{Behavioral results}

The distribution of averaged response proportions in the item recognition memory test (mean \pm SEM, collapsed across the first and

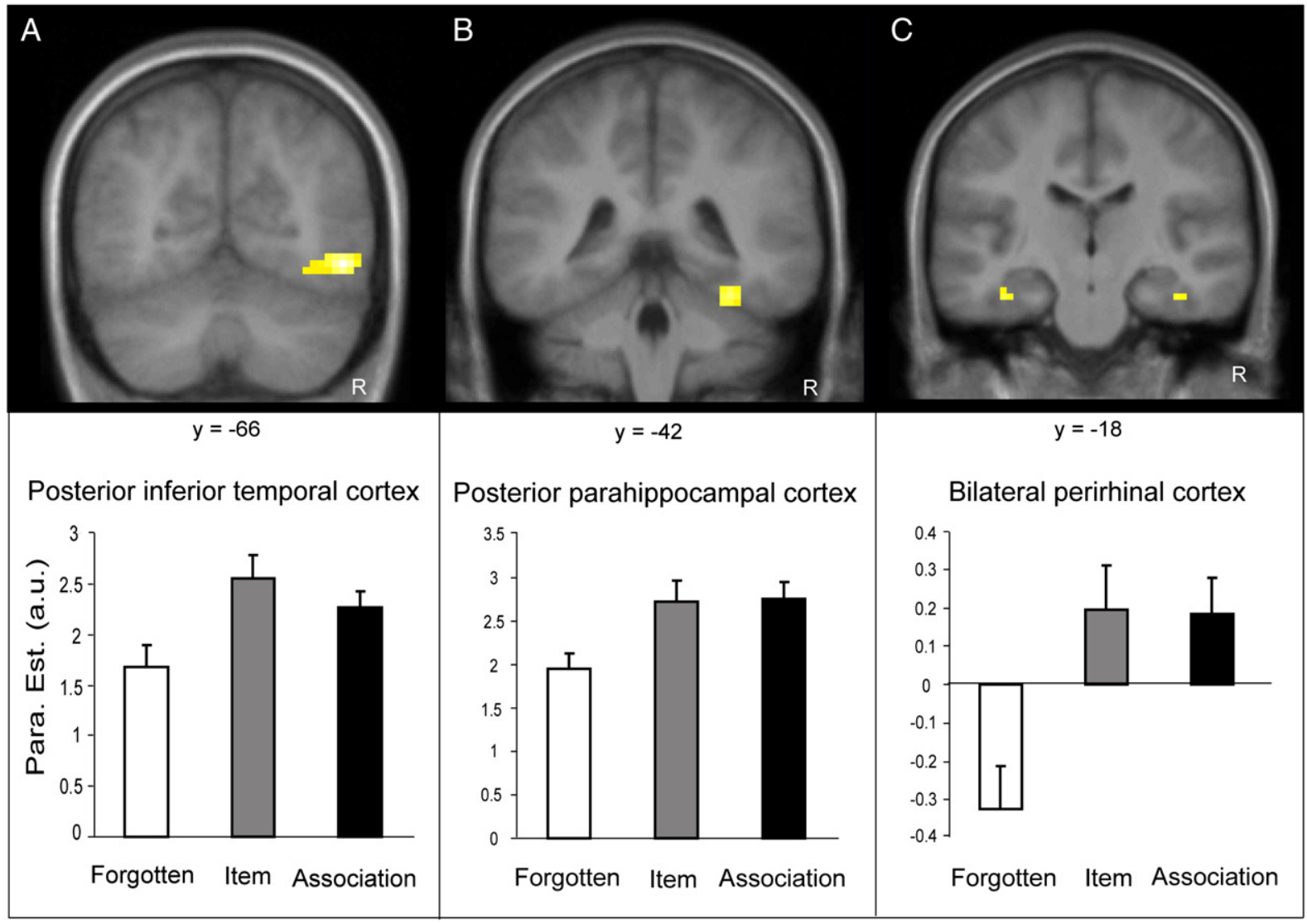

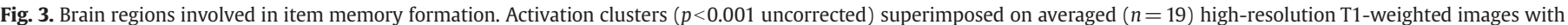

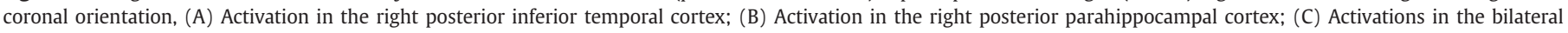

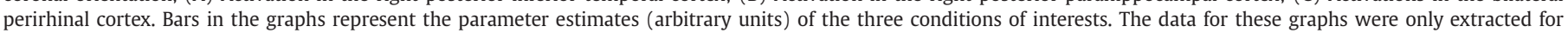
illustrative purposes and not for testing effects statistically. Notes: R, right. 
second event) are shown in Fig. 2. A two (stimulus type: old vs. new) by six (confidence rating: 6-point scale) repeated-measure ANOVA revealed an interaction between confidence rating and stimulus type $\left(F_{(5,90)}=59.92, p<0.001\right)$. Further paired-sample $t$ tests showed that the proportion of ' 6 ' and ' 5 ' ratings was significantly higher for old than new items (minimum $t_{(18)}=4.07, p<0.001$ ). The proportion of ' 4 ' ratings did not differ between old and new items $\left(t_{(18)}=-0.69\right.$, $p=0.49$ ). The proportion of ' 1 ', '2' and ' 3 ' ratings for old items was lower than for new ones (minimum $t_{(18)}=-4.48, p<0.001$ ). These results demonstrate successful discrimination between studied and unstudied items at all confidence levels except level 4. Associations were clearly retrieved above chance level (mean proportion of hits: 0.67; SEM: $0.08 ; t_{(18)}=8.63 ; p<0.001$ ), indicating successful memory formation for inter-item associations. Moreover, performance level for associative memory formation (mean of discriminability $d^{\prime}=0.68$; SEM: 0.07 ) and only item memory formation (mean of discriminability $d^{\prime}=0.86$; SEM: 0.12) appears similar $\left(t_{(18)}=-1.60, p=0.13\right)$, indicating a similar level of discriminability. Furthermore, when focusing on ' 5 ' and ' 6 ' confidence ratings, as done for the fMRI analysis, we observed no difference in the proportion of ' 6 ' ratings in the 'item' and the 'association' bins (mean proportion \pm SEM: $0.86 \pm 0.04$ vs. $0.90 \pm 0.04$ respectively; $t_{(18)}=1.33, p=0.20$ ), indicating again similar confidence levels in the two conditions of interest. However, the statistical comparison of $d^{\prime}$ might be problematic, because we used two different memory tests to measure item and associative memory. Thus, the estimates of $d^{\prime}$ may have different variances.

\section{Neuroimaging results}

To reveal brain activity related to item memory formation, we analyzed the data time-locked to the onset of the first event and contrasted trials that were related to a subsequent success in recognizing the item but failure to retrieve the association (i.e. 'item') with trials related to subsequent failure to remember both the item and the association (i.e. 'forgotten'). We found activated areas in the right fusiform gyrus potentially covered by parahippocampal cortex (local maximum at [33, - 42, -21]; cluster $p<0.05$ SVC), in the right posterior inferior temporal cortex (local maximum [48, $-66,-9]$; cluster $p<0.05$ corrected), and in the anterior parahippocampal gyrus bilaterally, presumably covered by perirhinal cortex (local maxima at $[-39,-18,-21]$, [42, -15 , -27 ]; with a less stringent threshold of $p<0.001$ uncorrected) (see Table 2; Fig. 3).

To identify brain regions related to successful associative memory formation, we analyzed the data time-locked to the onset of the second event and contrasted trials related to subsequent associative retrieval success (i.e. 'association') with trials related to subsequent success to recognize the item only (i.e. 'item'). In the MTL, we revealed an activation in the left hippocampus (local maximum at $[-33,-24,-21] ; p<0.05$ SVC). When masking this result with the hippocampal ROI, based on a normalized and averaged volume of individual hippocampal masks, 11 out of 15 voxels remained, confirming that this cluster clearly lies within the hippocampus. Outside the MTL, we found an activation linked to successful associative memory formation in the left inferior prefrontal cortex (local maxima at $[-48,30,-6]$ ), but with a less stringent threshold of $p<0.001$, uncorrected (see Table 2; Fig. 4). Given potential differences in brain activity between trials with confidence ratings of 5 and 6 , we conducted an additional analysis focusing on trials with a confidence rating of 6 . Although the number of trials appears insufficient for the 'item' bin, we still identified very similar left hippocampal activation, when contrasting

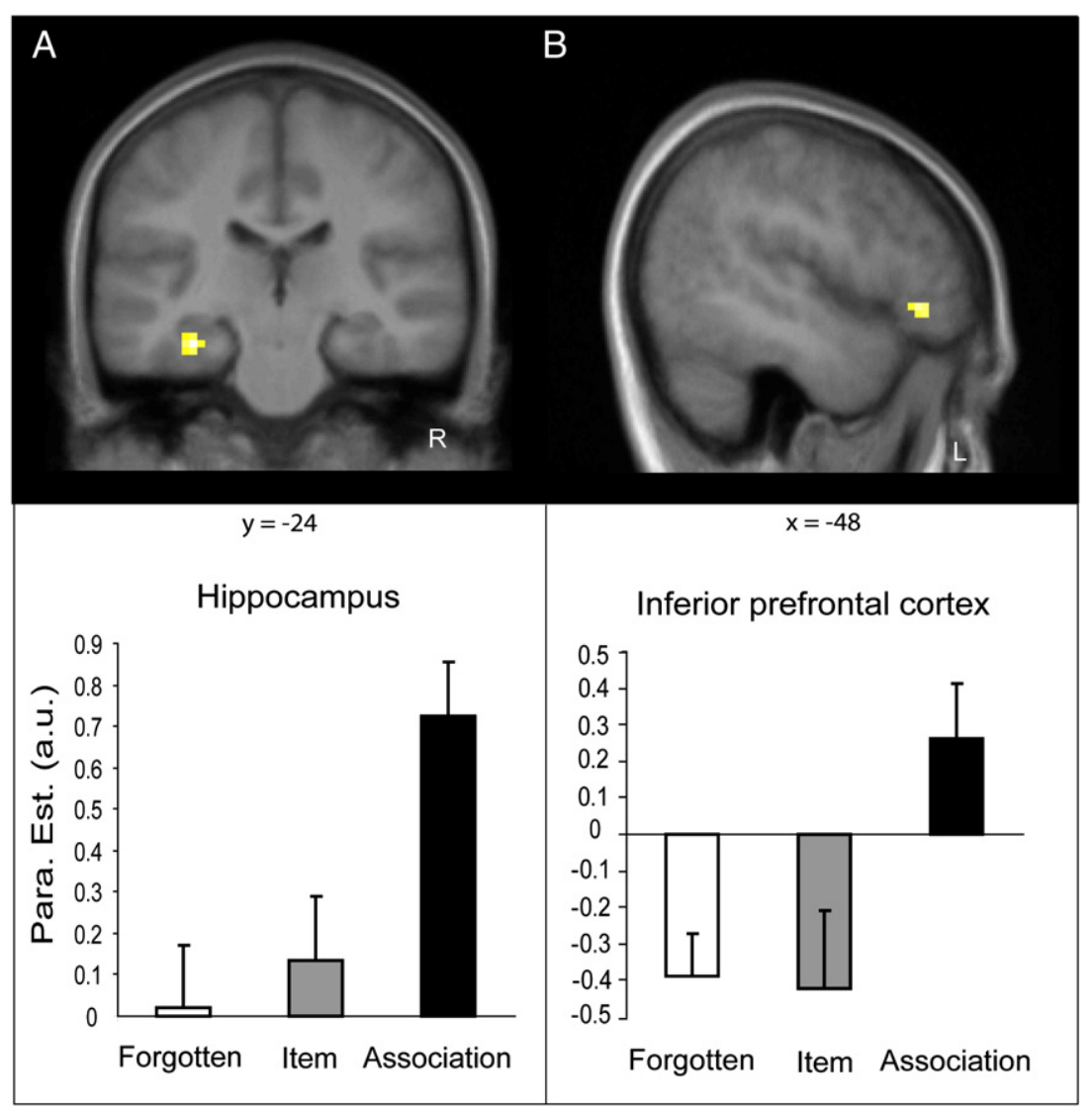

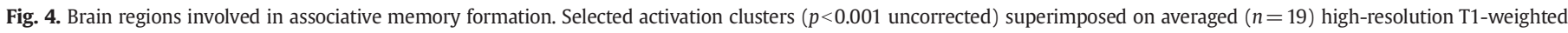

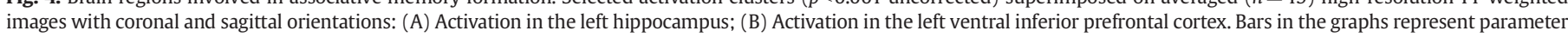

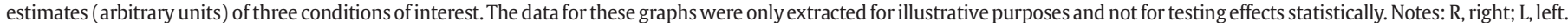


associative with item memory formation, just at a less stringent threshold ( $p<0.005$ uncorrected).

\section{Discussion}

Implementing a new task design in which processes underlying the formation of item memory and associative memory were separated in time and differences in memory strength between the two types of memory formation were potentially reduced, we found clear evidence for differences between hippocampal and parahippocampal contributions. While the hippocampal activity at encoding was associated only with subsequent associative memory retrieval, activity in the parahippocampal region predicted subsequent single item recognition. This dissociation is in line with several previous studies suggesting a two-component model of the MTL during encoding (Davachi et al., 2003; Ranganath et al., 2003; Chua et al., 2007; for reviews see Eichenbaum et al., 1994; Davachi, 2006). Given that subsequent confidence was similar for the two constituents in item and associative memories, differences in memory strength cannot readily explain the dissociation found. Thus, our data appear not supportive for a unitary MTL model proposed by Squire et al. (2007) in which different subcomponents act together in a complementary way while supporting both item and associative memory formation.

Our findings do indeed suggest that the MTL subregions play distinct roles in item and associative memory formation. The posterior inferior temporal cortex (including posterior fusiform gyrus) may initially contribute to the perceptual analysis of individual items (or objects) during encoding (Owen et al., 1996; Wagner et al., 1998; Brewer et al., 1998; Kao et al., 2005; Summerfield et al., 2006). Thereafter, a neocortical processing stream converges in the parahippocampal region where perceptual representations are unitized and thus can be transformed into meaningful representations for further usage (such as object identities and semantic meanings to facilitate associative memory formation) in the rhinal cortex (Nobre and McCarthy, 1995; Fernández et al., 2001; Levy et al., 2005), indicating that it is important for item memory formation (Davachi et al., 2003; Ranganath et al., 2003; Kao et al., 2005; Chua et al., 2007). This processing stream enables a-contextual encoding (such as objectrelated prototypical context) (Bar, 2004) and rapid familiarity detection when an item is already known by the very same process (Fernández and Tendolkar, 2006). Such a gradient accounts for the inferior temporal to perirhinal processing is consistent with some hierarchical models, which are mainly derived from studies in nonhuman primates, patients with intracranial event-related potential recordings as well as from neuroimaging studies in humans, suggesting that neocortical input of visual object features is sequentially processed in a posterior to anterior stream in order to transform the representational information from a perceptual level into a semantic one (Mishkin et al., 1983; Tanaka et al., 1991; Fernández et al., 2001; Vinckier et al., 2007). When the second event is perceived, relevant representational information converges further into the hippocampus, allowing representations of the first and the second event to be successfully bound together into a new association (Qin et al., 2007; Cheng et al., 2008; for review see Eichenbaum, 2004). The hippocampus is thought to be an optimal site to process these converging streams from the perirhinal cortex and the parahippocampal cortex that convey detailed information about item features and integrative information about the relevant context (Diana et al., 2007; Eichenbaum et al., 2007).

However, our data does not entirely exclude a single-process model for the MTL memory system which posits that recognition is based on a uni-dimensional value of memory strength. Despite similar performance levels, our contrast between item and associative memory might still be confounded by differences in memory strength. It may appear conceivable that a larger number of features were encoded for trials in which associative memory retrieval was successful as compared to trials in which only single items were later recognized. However, a monotonic increase in memory strength in subsequently forgotten trials over subsequent item memory trials to subsequent associative memory trials appears not in line with the data extracted from those activation areas. Only if one assumes a non-linear relationship between memory strength and the BOLD signal as proposed previously (Squire et al., 2007), our data could also be interpreted as being in line with a unitary MTL model. However, several studies have shown linear functions between neural activity and associated BOLD responses in a wide range of regions, but non-linear components occur at the extremes of the function and these non-linearities appear to be different in different brain structures (Boynton et al. 1996; Soltysik et al. 2004). Although there is no good reason to assume that different non-linear neuralBOLD functions occur within the MTL during normal memory tasks, there is to the best of our knowledge no study confirming the linearity of the neural-BOLD coupling in the MTL. Hence, we cannot exclude this alternative interpretation currently, but it appears not very likely.

The findings we obtained might be specifically depending on which kind of associations were formed and tested here. There is initial evidence that intra- and inter-item associations are supported by different MTL subregions (Mayes et al., 2004). The formation of intra-item associations appears to be related to processing in the parahippocampal region (Staresina and Davachi, 2006; Tendolkar et al., 2007), while inter-item associations are formed in the hippocampus (Mayes et al., 2004). The associations implemented here were created by arbitrary pairings of semantically (and perceptually) unrelated objects and thus, they can be clearly regarded as inter-item associations. Hence, our conclusion about the specific role of the hippocampus in associative memory formation is most likely limited to the formation of inter-item associations. Several neuroimaging studies have found consistently that the hippocampus was involved in storing new inter-item associations (such as word-pairs, face-name pairs) (Henke et al., 1999; Sperling et al., 2003; Kirwan and Stark, 2004; Prince et al., 2005; Chua et al., 2007; Qin et al., 2007). This interp|retation is consistent with the idea that the hippocampus is a general binding mechanism that associates separate items together (that might not be integrated and unified by the neocortex) to form a new association (Eichenbaum et al., 1994; Mayes et al., 2004).

An alternative interpretation of our hippocampal finding is related to the temporal delay between the two items to-be-associated. It is well accepted that the hippocampus is critically engaged in bridging discontinuities across time by its role in forming 'context units' and 'glueing' together of sequential items (Wallenstein et al., 1998; Eichenbaum, 2004). A recent neuroimaging study confirmed that the human hippocampus is indeed involved in sequence disambiguation by which overlapping sequences are kept separate and remembered correctly (Kumaran and Maguire, 2006). However, the presentation order was irrelevant for our task and only two items were paired together and thus our task can hardly be regarded as a sequence learning task. Nevertheless, the hippocampus was also found to be involved in 'discontinuity association' by bridging a delay between the constituents of word-pairs in anticipation of a semantic relatedness judgment (Luo and Niki, 2005), but this finding was not related to memory formation. Therefore, our present findings add to the aforementioned findings and suggest that the hippocampus is strongly involved in forming new inter-item associations between items that occur separated in time.

In addition to the hippocampus, the inferior prefrontal cortex was activated when associative memory formation was successful. The inferior prefrontal cortex may interact with the hippocampus and thus facilitate the formation of new associations by its role in 
recovering semantic information (Wagner et al., 2001) and in enabling semantic elaborative processes (Sperling et al., 2003; Jackson and Schacter, 2004; Prince et al., 2005; Ranganath and Blumenfeld, 2007). Nevertheless, this is not mutually exclusive from the view that the inferior prefrontal cortex involves generally in episodic memory formation including memories for items and associations as shown by numerous functional imaging studies (see reviews Fernández and Tendolkar, 2001; Wagner et al., 2001; Paller and Wagner, 2002; Simons and Spiers, 2003; Ranganath and Blumenfeld, 2007).

While this single study cannot exclude all alternative explanations for our findings like differences in strategic processing or differences in test formats for item and associative recognition memory our findings are most likely in line with dual process models of the MTL in which the parahippocampal region has a particular role in non-associative item memory and the hippocampus in memory for newly formed inter-item associations. This conclusion could be further strengthened by studies that confirm a linear neural-BOLD function in the MTL and by studies that control memory strength strictly quantitatively across item- and associative memory.

\section{Acknowledgments}

We thank Dr. Atsuko Takashima and Dr. Jianhui Wu for helpful discussions. We also thank all participants for their participation in this study.

\section{References}

Bar, M., 2004. Visual objects in context. Nat. Rev. Neurosci. 5, 617-629.

Boynton, G.M., Engel, S.A., Glover, G.H., Heeger, D.J., 1996. Linear systems analysis of functional magnetic resonance imaging in human V1. J. Neurosci. 16, 4207-4221.

Brett, M., Anton, J.L., Valabregue, R., Poline, J.B., 2002. Region of interest analysis using an SPM toolbox. Neurolmage 16 abstract 497 (available on CD-ROM).

Brewer, J.B., Zhao, Z., Desmond, J.E., Glover, G.H., Gabrieli, J.D., 1998. Making memories: brain activity that predicts how well visual experience will be remembered. Science 281, 1185-1187.

Brown, M.W., Aggleton, J.P., 2001. Recognition memory: what are the roles of the perirhinal cortex and hippocampus? Nat. Rev. Neurosci. 2, 51-61.

Cheng, D.T., Disterhoft, J.F., Power, J.M., Ellis, D.A., Desmond, J.E., 2008. Neural substrates underlying human delay and trace eyeblink conditioning. Proc. Natl. Acad. Sci. U.S.A. 105, 8108-8113.

Chua, E.F., Schacter, D.L., Rand-Giovannetti, E., Sperling, R.A., 2007. Evidence for a specific role of the anterior hippocampal region in successful associative encoding. Hippocampus 17, 1071-1080.

Davachi, L., Mitchell, J.P., Wagner, A.D., 2003. Multiple routes to memory: distinct temporal lobe processes build item and source memories. Proc. Natl. Acad. Sci. U. S. A. $100,2157-2162$.

Davachi, L., 2006. Item, context and relational episodic encoding in humans. Curr. Opin. Neurobiol. 16, 693-700.

Diana, R.A., Yonelinas, A.P., Ranganath, C., 2007. Imaging recollection and familiarity in the medial temporal lobe: a three-component model. Trends Cogn. Sci. 11, 379-386.

Dickerson, B.C., Salat, D.H., Greve, D.N., Chua, E.F., Rand-Giovannetti, E., Rentz, D.M., Bertram, L., Mullin, K., Tanzi, R.E., Blacker, D., Albert, M.S., Sperling, R.A., 2005. Increased hippocampal activation in mild cognitive impairment compared to normal aging and AD. Neurology 65, 404-411.

Eichenbaum, H., Otto, T., Cohen, N.J., 1994. Two functional components of the hippocampal memory system. Behav. Brain Sci. 17, 449-517.

Eichenbaum, H., 2004. Hippocampus: cognitive processes and neural representations that underlie declarative memory. Neuron 44, 109-120.

Eichenbaum, H., Yonelinas, A., Ranganath, C., 2007. The medial temporal lobe and recognition memory. Annu. Rev. Neurosci. 30, 123-152.

Fernández, G., Heitkemper, P., Grunwald, T., Van Roost, D., Urbach, H., Pezer, N., Lehnertz, K., Elger, C.E., 2001. Inferior temporal stream for word processing with integrated mnemonic function. Hum. Brain Mapp. 14, 251-260.

Fernández, G., Tendolkar, I., 2001. Integrated brain activity in medial temporal and prefrontal areas predicts subsequent memory performance: human declarative memory formation at the system level. Brain Res. Bull. 55, 1-9.

Fernández, G., Tendolkar, I., 2006. The rhinal cortex: 'gatekeeper' of the declarative memory system. Trends Cogn. Sci. 10, 358-362.

Friston, K., Holmes, A.P., Worsley, K., Poline, J., Frackowiak, R., 1995. Statistical parametric maps in functional imaging: a general linear approach. Hum. Brain Mapp. 2, 189-210.

Gonsalves, B.D., Kahn, I., Curran, T., Norman, K.A., Wagner, A.D., 2005. Memory strength and repetition suppression: multimodal imaging of medial temporal cortical contributions to recognition. Neuron 47, 751-761.
Hales, J.B., Israel, S.L., Swann, N.C., Brewer, J.B., 2009. Dissociation of frontal and medial temporal lobe activity in maintenance and binding of sequentially presented paired associates. J. Cog. Neurosci. doi:10.1162/jocn.2009.21096.

Hannula, D.E., Ranganath, C., 2008. Medial temporal lobe activity predicts successful relational memory binding. J. Neurosci. 28, 116-124

Henke, A., Weber, B., Kneifel, S., Wieser, H.G., Buck, A., 1999. Human hippocampus associates information in memory. Proc. Natl. Acad. Sci. U. S. A. 96, 5884-5889.

Henson, R.N., 2005. A mini-review of fMRI studies of human medial temporal lobe activity associated with recognition memory. Q. J. Exp. Psychol. B 58, 340-360.

Jackson, O., Schacter, D.L., 2004. Encoding activity in anterior medial temporal lobe supports subsequent associative recognition. Neurolmage 21, 456-462.

Kao, Y.C., Davis, E.S., Gabrieli, J.D., 2005. Neutral correlates of actual and predicted memory formation. Nat. Neurosci. 8, 1776-1783.

Kirwan, C.B., Stark, C.E., 2004. Medial temporal lobe activation during encoding and retrieval of novel face-name pairs. Hippocampus 14, 919-930.

Kumaran, D., Maguire, E.A., 2006. The dynamics of hippocampal activation during encoding of overlapping sequences. Neuron 49, 617-629.

Levy, D.A., Shrager, Y., Squire, L.R., 2005. Intact visual discrimination of complex and feature-ambiguous stimuli in the absence of perirhinal cortex. Learn. Mem.12,61-66.

Luo, J., Niki, K., 2005. Does hippocampus associate discontiguous events? Evidence from event related fMRI. Hippocampus 15, 141-148.

Mayes, A.R., Holdstock, J.S., Isaac, C.L., Montaldi, D., Grigor, J., Gummer, A., Cariga, P., Downes, J.J., Tsivilis, D., Gaffan, D., Gong, Q., Norman, K.A., 2004. Associative recognition in a patient with selective hippocampal lesions and relatively normal item recognition. Hippocampus 14, 763-784.

Miller, S.L., Celone, K., DePeau, K., Diamond, E., Dickerson, B.C., Rentz, D., Pihlajamäki, M. Sperling, R.A., 2008. Age-related memory impairment associated with loss of parietal deactivation but preserved hippocampal activation. Proc. Natl. Acad. Sci. U.S.A. 105, 2181-2186.

Mishkin, M., Ungerleider, L.G., Macko, K.A., 1983. Object vision and spatial vision: two cortical pathways. Trends Neurosci. 6, 414-417.

Montaldi, D., Spencer, T.J., Roberts, N., Mayes, A.R., 2006. The neural system that mediates familiarity memory. Hippocampus $16,504-520$.

Murray, L.J., Ranganath, C., 2007. The dorsolateral prefrontal cortex contributes to successful relational memory encoding. J. Neurosci. 27, 5515-5522.

Nobre, A.C., McCarthy, G., 1995. Language-related field potentials in the anterior-media temporal lobe. II. Effects of word type and semantic priming. J. Neurosci. 15, 1090-1098.

Owen, A.M., Milner, B., Petrides, M., Evans, A.C., 1996. Memory for object features versus memory for object location: a positron-emission tomography study of encoding and retrieval processes. Proc. Natl. Acad. Sci. U. S. A. 93, 9212-9217.

Paller, K.A., Wagner, A.D., 2002. Observing the transformation of experience into memory. Trends Cogn. Sci. 6, 93-102.

Prince, S.E., Daselaar, S.M., Cabeza, R., 2005. Neural correlates of relational memory: successful encoding and retrieval of semantic and perceptual associations. J. Neurosci. $25,1203-1210$

Pruessner, J.C., Li, L.M., Serles, W., Pruessner, M., Collins, D.L., Kabani, N., Lupien, S. Evans, A.C., 2000. Volumetry of hippocampus and amygdala with high-resolution MRI and three-dimensional analysis software: minimizing the discrepancies between laboratories. Cereb. Cortex 10, 433-442.

Pruessner, J.C., Köhler, S., Crane, J., Pruessner, M., Lord, C., Byrne, A., Kabani, N. Collins, D.L., Evans, A.C., 2002. Volumetry of temporopolar, perirhinal, entorhinal and parahippocampal cortex from high-resolution MR images: considering the variability of the collateral sulcus. Cereb. Cortex 12, 1342-1453.

Qin, S.Z., Piekema, C., Petersson, K.M., Han, B.X., Luo, J., Fernández, G., 2007. Probing the transformation of discontinuous associations into episodic memory: an eventrelated fMRI study. NeuroImage 38, 212-222.

Ranganath, C., Blumenfeld, R.S., 2007. Prefrontal cortex and human memory: an integrated account of results from neuropsychological and neuroimaging studies of working- and long-term memory. In: Eichenbaum, H. (Ed.), Learning and Memory: A Comprehensive Reference. Elsevier, Oxford, UK.

Ranganath, C., Yonelinas, A.P., Cohen, M.X., Dy, C.J., Tom, S.M., D'Esposito, M., 2003. Dissociable correlates of recollection and familiarity within the medial temporal lobes. Neuropsychologia 42, 2-13.

Rutishauser, U., Mamelak, A.N., Schuman, E.M., 2006. Single-trial learning of nove stimuli by individual neurons of the human hippocampus-amygdala complex. Neuron 49, 805-813.

Shrager, Y., Kirwan, C.B., Squire, L.R., 2008. Activity in both hippocampus and perirhinal cortex predicts the memory strength of subsequently remembered information. Neuron 59, 547-553.

Simons, J.S., Spiers, H.J., 2003. Prefrontal and medial temporal lobe interactions in long-term memory. Nat. Rev. Neurosci. 4, 637-648.

Soltysik, D.A., Peck, K.K., White, K.D., Crosson, B., Briggs, R.W., 2004. Comparison of hemodynamic response nonlinearity across primary cortical areas. NeuroImage 22 1117-1127.

Sperling, R.A., Greve, D., Dale, A., Killiany, R., Holmes, J., Rosas, H.D., Cocchiarella, A., Firth, P., Rosen, B., Lake, S., Lange, N., Routledge, C., Albert, M.S., 2002. Functional MRI detection of pharmacologically induced memory impairment. Proc. Natl. Acad. Sci. U. S. A. 99, 455-460.

Sperling, R.A., Chua, E., Cocchiarella, A., Giovannetti, E.R., Poldrack, R., Schacter, D.L., Albert, M.S., 2003. Putting names to faces: successful encoding of associative memories activates the anterior hippocampal formation. NeuroImage 20, $1400-1410$.

Squire, L.R., Wixted, J.T., Clark, R.E., 2007. Recognition memory and the medial temporal lobe: a new perspective. Nat. Rev. Neurosci. 8, 872-883.

Staresina, B.P., Davachi, L, 2006. Differential encoding mechanisms for subsequent associative recognition and free recall. J. Neurosci. 26, 9162-9172. 
Staresina, B.P., Davachi, L., 2008. Selective and shared contributions of the hippocampus and perirhinal cortex to episodic item and associative encoding. J. Cogn. Neurosci. $20,1478-1489$.

Summerfield, C., Greene, M., Wager, T., Egner, T., Hirsch, J., Mangels, J., 2006. Neocortica connectivity during episodic memory formation. PLoS Biol. 4, e128.

Tanaka, K., Saito, H., Fukada, Y., Moriya, M., 1991. Coding visual images of objects in the inferotemporal cortex of the macaque monkey. J. Neurophysiol. 66, 170-189.

Tendolkar, I., Arnold, J., Petersson, K.M., Weis, S., Brockhaus-Dumke, A., van Eijndohoven, P., Buitelaar, J., Fernández, G., 2007. Probing the neural correlates of associative memory formation: a parametrically analyzed event-related functional MRI study. Brain Res. 1142, 159-168.

Tendolkar, I., Arnold, J., Petersson, K.M., Weis, S., Brockhaus-Dumke, A., van Eijndhoven, P., Buitelaar, J., Fernández, G., 2008. Contributions of the medial temporal lobe to declarative memory retrieval: manipulating the amount of contextual retrieval. Learn. Mem. 15, 611-617.

Tulving, E., 1983. Elements of Episodic Memory. Oxford Univ. Press, New York.

Tulving, E., 2002. Episodic memory: from mind to brain. Annu. Rev. Psychol. 53, 1-25

Uncapher, M.R., Otten, L.J., Rugg, M.D., 2006. Episodic encoding is more than the sum of its parts: an fMRI investigation of multifeatural contextual encoding. Neuron 52, 547-556.
Vinckier, F., Dehaene, S., Jobert, A., Dubus, J.P., Sigman, M., Cohen, L., 2007. Hierarchical coding of letter strings in the ventral stream: dissecting the inner organization of the visual word-form system. Neuron 55, 143-156.

Wagner, A.D., Schacter, D.L., Rotte, M., Koutstaal, W., Maril, A., Dale, A.M., Rosen, B.R., Buckner, R.L., 1998. Building memories: remembering and forgetting of verbal experiences as predicted by brain activity. Science 281, 1188-1191.

Wagner, A.D., Pare-Blagoev, E.J., Clark, J., Poldrack, R.A., 2001. Recovering meaning: left prefrontal cortex guides controlled semantic retrieval. Neuron 31, 329-338.

Wallenstein, G.V., Eichenbaum, H., Hasselmo, M.E., 1998. The hippocampus as an associator of discontiguous events. Trends Neurosci. 21, 317-323.

Weis, S., Specht, K., Klaver, P., Tendolkar, I., Willmes, K., Ruhlmann, J., Elger, C.E., Fernández, G., 2004. Process dissociation between contextual retrieval and item recognition. NeuroReport 15, 2729-2733.

Worsley, K.J., Marrett, S., Neelin, P., Vandal, A.C., Friston, K.J., Evans, A.C., 1996. A unified statistical approach for determining significant signals in images of cerebral activation. Hum. Brain Mapp. 4, 58-73.

Yonelinas, A.P., Kroll, N.E., Quamme, J.R., Lazzara, M.M., Sauvé, M.J., Widaman, K.F., Knight, RT, 2002. Effects of extensive temporal lobe damage or mild hypoxia on recollection and familiarity. Nat. Neurosci. 5, 1236-1241. 